ARTICLE

DOI: $10.1038 / \mathrm{s} 41467-018-03368-9$

\title{
Studying an antiaromatic polycyclic hydrocarbon adsorbed on different surfaces
}

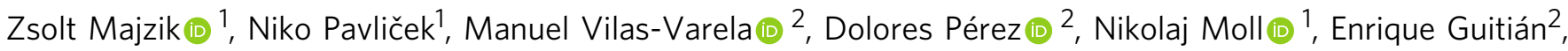
Gerhard Meyer ${ }^{1}$, Diego Peña (10 ${ }^{2}$ \& Leo Gross (10 ${ }^{1}$

Antiaromatic and open-shell molecules are attractive because of their distinct electronic and magnetic behaviour. However, their increased reactivity creates a challenge for probing their properties. Here, we describe the on-surface and in-solution generation and characterisation of a highly reactive antiaromatic molecule: indeno[1,2-b]fluorene (IF). In solution, we generated IF by $\mathrm{KI}$-induced dehalogenation of a dibromo-substituted precursor molecule and found that IF survives for minutes at ambient conditions. Using atom manipulation at low temperatures we generated IF on $\mathrm{Cu}(111)$ and on bilayer $\mathrm{NaCl}$. On these surfaces, we characterised IF by bond-order analysis using non-contact atomic force microscopy with COfunctionalised tips and by orbital imaging using scanning tunnelling microscopy. We found that the closed-shell configuration and antiaromatic character predicted for gas-phase IF are preserved on the $\mathrm{NaCl}$ film. On $\mathrm{Cu}(111)$, we observed significant bond-order reorganisation within the s-indacene moiety because of chemisorption, highlighting the importance of molecule surface interactions on the $\pi$-electron distribution.

\footnotetext{
${ }^{1}$ IBM Research - Zurich, 8803 Rüschlikon, Switzerland. ${ }^{2}$ Centro Singular de Investigación en Química Biolóxica e Materiais Moleculares (CiQUS), Departamento de Química Orgánica, Universidade de Santiago de Compostela, 15782 Santiago de Compostela, Spain. Correspondence and requests for materials should be addressed to Z.M. (email: maj@zurich.ibm.com) or to D.Pña. (email: diego.pena@usc.es)
} 
A romaticity is one of the most relevant, and intriguing, concepts in chemistry ${ }^{1,2}$. In 1931, Hückel suggested his well-known rule to explain the extra stability of planar monocyclic molecules that contain $[4 n+2] \pi$-electrons in a conjugated system ${ }^{3,4}$. With some limitations, the concept was later extended to polycyclic conjugated hydrocarbons (PCHs), based on the number of Clar sextets ${ }^{5}$, or the presence of conjugated circuits with $[4 n+2] \pi$-electrons within a particular structure $^{6}$. In 1967, Breslow introduced the term antiaromaticity as the inverse of aromaticity, in order to explain the destabilisation of molecules with $[4 n] \pi$-electrons in a cyclic conjugated system ${ }^{7}$. Among the PCHs with antiaromatic character ${ }^{8}$, indeno $[1,2-b]$ fluorene (IF) is a remarkable example (Fig. 1) $)^{9,10}$. Compared with pentacene $(\mathbf{1})$, the most prominent p-type organic semiconductor with five linearly fused six-membered rings and $22 \pi$-electrons in its aromatic conjugate circuit $([4 n+2], n=5)$, IF presents a 6-5-6-5-6 fused-ring motif and a formally antiaromatic $20 \pi$-electron conjugate system $([4 n] n=5)$. The IF closed-shell configuration with a central eight $\pi$-electron paraquinodimethane core (in red, Fig. 1) and two Clar sextets (in blue) might be in resonance with the open-shell diradical configuration with three sextets ${ }^{11,12}$. As a result, unsubstituted IF is presumed to be an extremely reactive $\mathrm{PCH}$ and in fact has never been synthesised or even detected to date.

In contrast, in recent years large effort have been devoted to the preparation of substituted-IF derivatives that are stable enough to be isolated and studied in detail ${ }^{13-17}$. These antiaromatic PCHs have received special attention because of their distinct electron accepting, n-type semiconducting behaviour, based on their easy reduction to the corresponding aromatic dianions ${ }^{13,18}$. This feature, together with the narrow highest occupied molecular orbital (HOMO)-lowest unoccupied molecular orbital (LUMO) gap, make IF derivatives promising organic photoelectronic materials $^{19}$. The high reactivity expected for unsubstituted IF implies a challenge for probing its structural and electrical properties. An interesting fact if that the parent structure of one of the most promising $\mathrm{PCH}$ cores for electronic devices has not yet been investigated $^{20}$.

Advances in atomic force microscopy (AFM), particularly in resolving ${ }^{21}$ and modifying the structure of molecules at the atomic scale, have opened new routes in the chemistry of highly reactive compounds ${ }^{22-30}$. AFM with functionalised tips has been used to identify and characterise individual molecules ${ }^{31-34}$. One important aspect is the bond order, which can be resolved directly with AFM by comparing the apparent length and contrast of individual bonds within the molecule ${ }^{31}$, which has already been applied for the on-surface characterisation of biphenylenes ${ }^{35}$ and highly reactive molecules such as arynes ${ }^{29}$ or diradicals ${ }^{30}$.

Here we present the generation and characterisation of an antiaromatic $\mathrm{PCH}$, i.e. IF comprising a $\pi$-system with 20 electrons. In-solution IF was generated by iodide-induced decomposition of dibromo-substituted precursor 2, whereas on-surface IF was obtained by tip-induced dehydrogenation of the polycyclic hydrocarbon 3. We found that IF survives for a few minutes in solution at ambient conditions. Using AFM and STM at low temperature and in ultra-high vacuum, we found that on bilayer $\mathrm{NaCl}$ on $\mathrm{Cu}(111)$ (denoted as 2 monolayers or $2 \mathrm{ML} \mathrm{NaCl}$ ) IF preserves the closed-shell character predicted for the free molecule, whereas on $\mathrm{Cu}(111)$ the electronic configuration is significantly altered because of strong chemisorption to the surface.

\section{Results}

In-solution synthesis and lifetime of IF. We explored the insolution generation of IF by KI-induced decomposition of 6,12dibromo-6,12-dihydro indeno[1,2-b]fluorene (2) (Fig. 1). It is
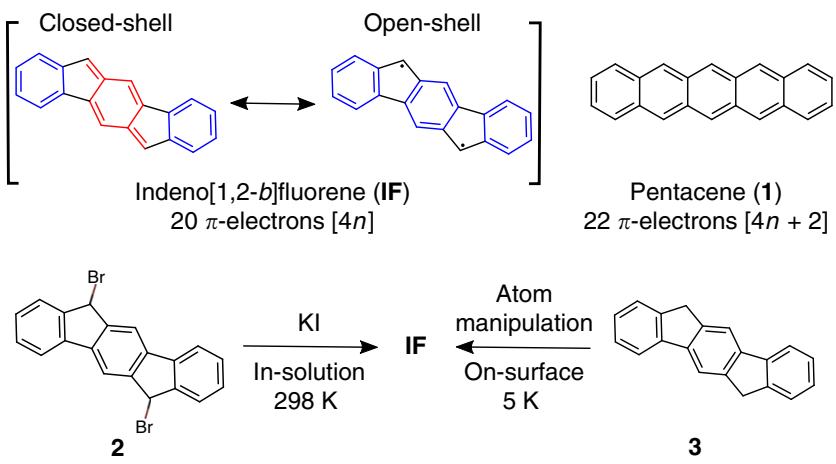

Fig. 1 Molecular structures of indeno[1,2-b]fluorene (IF) and related compounds. The central eight $\pi$-electron para-quinodimethane core of IF is shown in red, the six $\pi$-electron Clar sextets are shown in blue

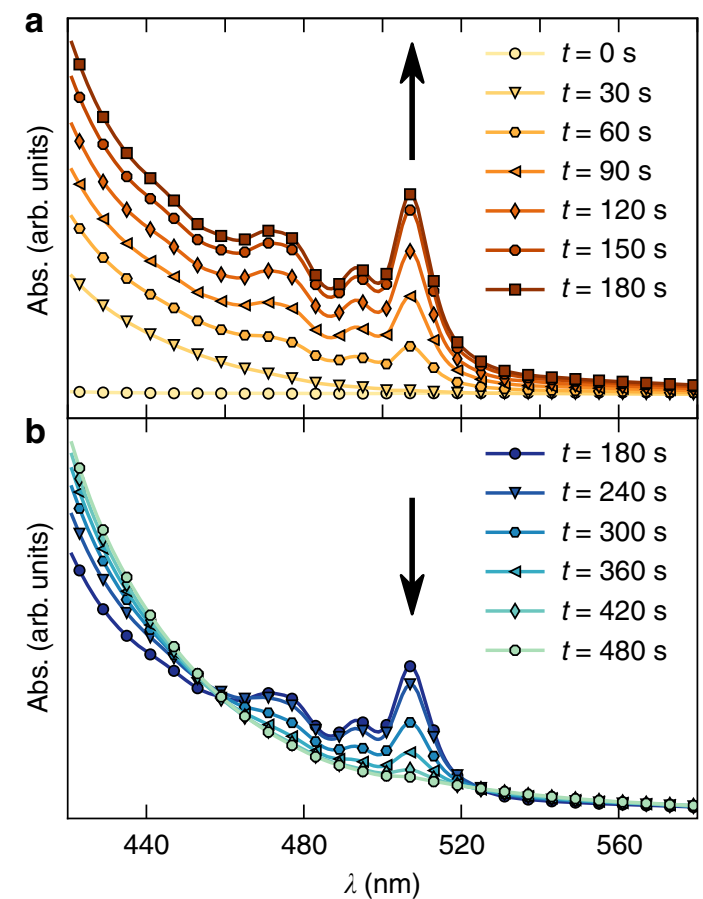

Fig. $2 \mathrm{KI}$-induced dehalogenation of $\mathbf{2}$. The reaction was monitored by UV spectroscopy measured in a 1:1 mixture of THF: $\mathrm{CH}_{3} \mathrm{CN}$ at $20^{\circ} \mathrm{C}$. The first measurement $(t=0)$ was taken before the addition of KI. a Shows the time before the peak at $507 \mathrm{~nm}$ has reached its maximum and $\mathbf{b}$ after

well known that highly reactive ortho-quinodimethane can be generated by iodide-induced debromination from the corresponding precursors ${ }^{36,37}$. Based on these precedents, we synthesised compound 2 (see Supplementary Methods for details) to study the generation of IF at room temperature by treatment with $\mathrm{KI}$ in a mixture of THF: $\mathrm{CH}_{3} \mathrm{CN}$ (1:1). The reaction was monitored by means of UV spectroscopy (Fig. 2). Before the KI treatment $(t=0)$, we did not observe any absorbance between 440 and $560 \mathrm{~nm}$. However, immediately after adding KI, we observed a group of signals appearing between 460 and $520 \mathrm{~nm}$ $\left(\lambda_{\max }\right.$ ca. $\left.507 \mathrm{~nm}\right)$, which are characteristic of the IF chromophore. Similar UV spectra were reported for 6,12-dimesityl-substituted IF, where there is a little electronic communication between the $\pi$-systems of the orthogonal mesityl groups and IF is very small ${ }^{18}$. The detected optical gap $E_{\text {gap }}$ and the position of the absorption peak $\lambda_{\max }$ in our experiment are nearly identical with those of 6,12-dimesityl-substituted IF reported before (Table 1) ${ }^{18}$. The peak at $507 \mathrm{~nm}$ reaches its maximum intensity after $180 \mathrm{~s}$ of 


\section{Table 1 Computational and optical data}

\begin{tabular}{|llllllll} 
& \multicolumn{3}{c}{ Computational } & \multicolumn{3}{c}{ Optical } \\
\cline { 2 - 4 } \cline { 7 - 8 } Compound & $\boldsymbol{E}_{\text {HOMO }}$ & $\boldsymbol{E}_{\text {LUMO }}$ & $\boldsymbol{E}_{\text {gap }}$ & & $\lambda_{\text {max }}$ & $\lambda_{\text {edge }}$ & $\boldsymbol{E}_{\text {gap }}$ \\
\hline $\mathbf{2}$ & -5.98 & -2.19 & 3.79 & & 329 & 345 & 3.59 \\
$\mathbf{I F}$ & -5.42 & -3.17 & 2.25 & & 507 & 534 & 2.33 \\
6,12-Dimesityl-IF & -5.32 & -3.15 & 2.17 & & $516^{18}$ & $541^{18}$ & $2.29^{18}$ \\
\hline
\end{tabular}

Hybrid functional HSE (Heyd, Scuseria and Ernzerhof) $)^{54,55}$ with a mixing coefficient of 0.3 was applied for computational calculations. Wavelengths $\lambda_{\max }$ and $\lambda_{\text {edge }}$ are in $\mathrm{nm}$ and energies $E_{\text {HOMO, }} E_{\text {LUMO }}$ and $E_{\text {gap }}$ are in $\mathrm{eV}$

KI treatment; then it starts to vanish owing to the short lifetime of highly reactive IF (Supplementary Discussion).

On-surface generation and characterisation of IF. With the idea to increase the lifetime of IF and explore its aromaticity, we deposited compound 3 in ultra-high vacuum at low temperature on $\mathrm{Cu}(111)$ partly covered by bilayer $\mathrm{NaCl}$ islands. To dehydrogenate 3, we followed a similar procedure as that for the dehydrogenation of triangulene precursors ${ }^{38}$. A Cu-terminated tip was positioned above the centre of a precursor molecule 3 at a tip height corresponding to an STM setpoint of $V=0.1 \mathrm{~V}$ and $I$ $=1 \mathrm{pA}$. At the opened-feedback loop the tip was retracted by $4-8$ $\AA$ to limit the tunnelling current to a few picoamperes at elevated biases, then the sample voltage $V$ was increased for $2 \mathrm{~s}$. Typically, a sudden change in the tunnelling current occurred within two seconds at biases above $3.5 \mathrm{~V}$, indicating a manipulation event $^{24,27,28,38,39}$. The threshold for dehydrogenation $(3.5 \mathrm{~V})$ is consistent with previous experiments to remove a single $\mathrm{H}$ from doubly benzylic $\mathrm{CH}_{2}$ groups ${ }^{38}$ and with the dissociation energy of a C-H bond within the $\mathrm{CH}_{2}$ group of fluorene $(80 \mathrm{~kJ} / \mathrm{mol}$ or 3.47 $\mathrm{eV})^{40}$. On $\mathrm{Cu}(111)$, two hydrogens were removed sequentially, giving rise to two distinct steps in the current. On $2 \mathrm{ML} \mathrm{NaCl}$, IF formed directly without the formation of a stable intermediate indicating concerted dehydrogenation (see Supplementary Fig. 6 and its discussion for more details about the on-surface generation of IF).

Figure 3 shows constant-height AFM images before and after dehydrogenation on both surfaces and also of the intermediate $\mathbf{3}^{\prime}$ after dissociation of only one $\mathrm{H}$ on $\mathrm{Cu}(111)$. AFM images were taken with a $\mathrm{CO}$-functionalised tip at zero bias $(V=0)$. Height offsets $\Delta z$ are denoted with respect to an STM setpoint of $I=1$ $\mathrm{pA}$ at $V=0.1 \mathrm{~V}$ above the respective substrate surface. A positive $\Delta z$ sign corresponds to an increase in the tip-sample separation. The structure of the precursor molecule adsorbed on $\mathrm{Cu}(111)$ was resolved using $\Delta z=-1.3 \AA$. After complete dehydrogenation, molecular resolution was acquired at typically $\Delta z=-2.5 \AA$, indicating a significantly reduced adsorption height. In contrast, on $2 \mathrm{ML} \mathrm{NaCl}$ we observed atomic resolution of 3 at $\Delta z=1.7$ and of IF at $1.5 \AA$, suggesting only a minor change in the adsorption height due to dehydrogenation on $\mathrm{NaCl}$. We quantified the change in the adsorption height with $\Delta f(z)$ spectroscopy using $z^{*}$, the tip height of which $\Delta f(z)$ is minimal, as a measure of the relative adsorption height (Fig. 4$)^{28}$. The $z^{*}$ map acquired on $\mathrm{Cu}$ ( $2 \mathrm{ML} \mathrm{NaCl})$ shows that IF adsorbs $0.94 \AA(0.18 \AA)$ closer to the surface than 3 . In addition, the $z^{*}$ maps indicate that IF adsorbs planarly on $\mathrm{NaCl}$. On $\mathrm{Cu}(111)$ we observed a slight increase in the adsorption height at the outer benzene rings than at the molecular centre, similar to pentacene (1) adsorbed on $\mathrm{Cu}(111)^{21,28}$.

In solution, the IF core can be easily transformed into [ $4 n+2]$ aromatic dication ( $18 \pi$-electrons) or dianion ( $22 \pi$-electrons) by oxidation or reduction reactions, respectively ${ }^{13,18}$. On $2 \mathrm{ML}$ $\mathrm{NaCl}$, the charge state of the adsorbed molecule is governed by

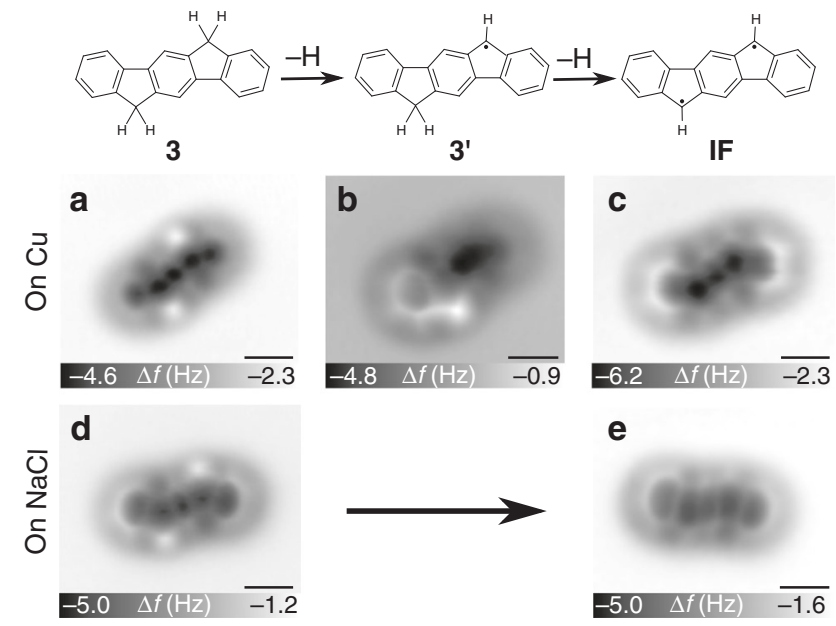

Fig. 3 On-surface generation of indeno[1,2-b]fluorene (IF). 6,12Dihydroindeno[1,2-b]fluorene (3) was dehydrogenated with bias pulses typically between 3.5 and $4.3 \mathrm{~V}$. a-e Constant-height AFM images of the precursor molecule $\mathbf{3}(\mathbf{a}, \mathbf{d})$, of radical $\mathbf{3}^{\prime}(\mathbf{b})$ and IF $(\mathbf{c}, \mathbf{e})$ measured with a CO tip at $V=0$ on $\mathrm{Cu}(111)$ (a-c) and on $2 \mathrm{ML} \mathrm{NaCl}(\mathbf{d}, \mathbf{e})$, respectively. Images were taken at tip height offsets a $\Delta z=-1.3 \AA$, b $\Delta z=-2.0 \AA, \mathbf{c} \Delta z$ $=-2.5 \AA, \mathbf{d} \Delta z=1.7 \AA$ and $\mathbf{e} \Delta z=1.5 \AA$ with respect to the STM setpoint of $I=1.0 \mathrm{pA}$ and $V=0.1 \mathrm{~V}$ above the respective substrate. Scale bars, 500 $\mathrm{pm}$

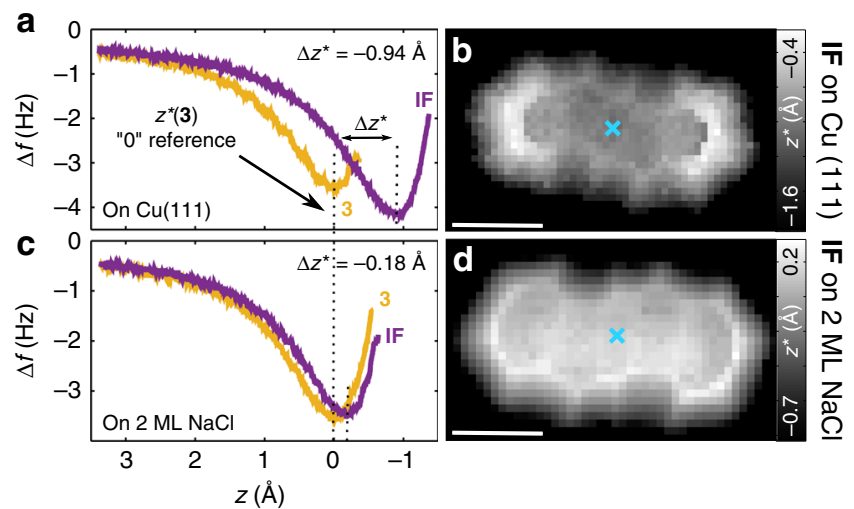

Fig. $4 \Delta f(z)$ spectroscopy. a, c $\Delta f(z)$ curves taken over the central benzene ring of $\mathbf{3}$ (yellow) and IF (purple) adsorbed on a $\mathrm{Cu}(111)$ and $\mathbf{c} 2 \mathrm{ML} \mathrm{NaCl}$. The $z$ scale is offset by $z^{\star}(\mathbf{3})$ for the respective surface to show $\mathbf{3}$ at $z=0$ as reference. $\mathbf{b}, \mathbf{d} z^{\star}$ maps of IF adsorbed on $\mathbf{b} \mathrm{Cu}(111)$ and $\mathbf{d} 2 \mathrm{ML} \mathrm{NaCl}$. At $z=-1.6 \AA$ on $\mathrm{Cu}(111)$ and $z=-0.7 \AA$ on $2 \mathrm{ML} \mathrm{NaCl}$ (black regions), the approach was aborted to avoid tip instabilities, and $z^{\star}$ was not reached above the bare surfaces. Scale bars, $500 \mathrm{pm}$

the work function of the surface and by the electron affinity level and the ionisation potential of the molecule ${ }^{41}$. We observed no interface state electron scattering at IF on $2 \mathrm{ML} \mathrm{NaCl}$, demonstrating that the molecule remains neutral and therefore antiaromatic on $2 \mathrm{ML} \mathrm{NaCl}^{42}$.

To learn about the contributions of the open- and closed-shell resonant structures presented in Fig. 1, we probed the orbital configuration of IF on $\mathrm{NaCl}$ and carried out spin-polarised DFT calculations with a first-order perturbative correction $\left(G_{0} W_{0}\right)$. For the calculations, we considered the molecule in its open-shell and closed-shell configurations (Methods). Their quasiparticle energies are shown in Fig. 5a. The zero of the energy scale has been adjusted to match the experimentally determined work function of $2 \mathrm{ML} \mathrm{NaCl}$ on $\mathrm{Cu}(111)(\Phi=4.0 \mathrm{eV})^{43}$. Based on the level alignments, IF is predicted to remain uncharged in both cases 
a

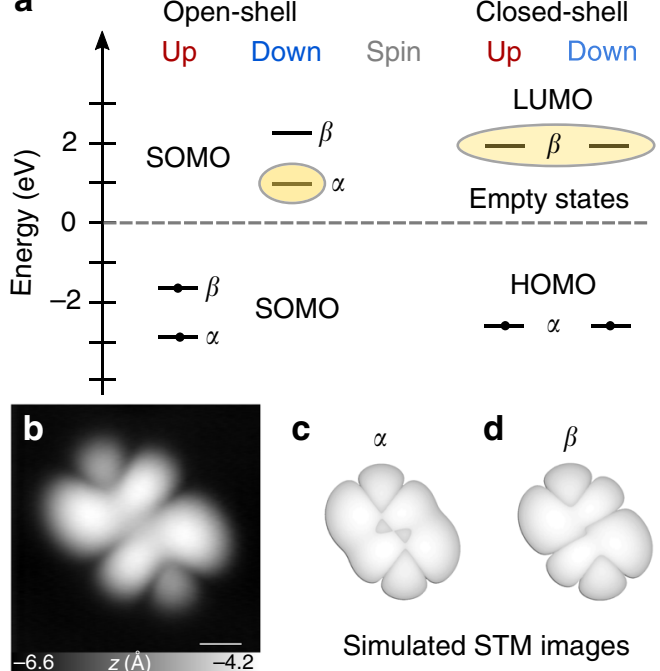

Fig. 5 Spin-polarised DFT calculations. a Energy-level diagram obtained from DFT calculations carried out with the $G_{0} W_{0}$ approximation. Marked energy levels correspond to the lowest-laying unoccupied states. $\mathbf{b}$

Constant-current STM image of IF taken with a metal tip at the energy corresponding to the negative ion resonance (NIR) $(V=1.0 \mathrm{~V}$ and $I=1.0$ $\mathrm{pA})$. c, d Simulated STM images of orbitals $\alpha(\mathbf{c})$ and $\beta(\mathbf{d})$. Scale bar, 500 $\mathrm{pm}$

considered. $\alpha$ and $\beta$ are the frontier molecular orbitals of IF. In the closed-shell configuration, $\alpha$ is fully occupied (closed) and is the highest occupied molecular orbital, whereas $\beta$ is the lowest unoccupied molecular orbital. Note that non-polarised energy minimisation also leads to the closed-shell configuration. In the open-shell configuration, $\alpha$ and $\beta$ are non-degenerate singly occupied molecular orbitals (SOMOs). We imaged the negative ion resonance (NIR) at positive sample bias, with its onset at $V=$ $1.0 \mathrm{~V}$ (Fig. 5b). We were unable to image the positive ion resonance (PIR) because the molecule started to move at increased negative biases before the bias voltage reached the onset of the PIR. Because IF is neutral, the NIR is attributed to electron tunnelling into the lowest-lying empty state(s) of the free molecule $^{42}$. In the closed-shell case, the NIR is expected to closely resemble the shape of the LUMO (orbital $\beta$ ), whereas in the openshell case, the NIR is related to the empty spin-down channel of the $\alpha$ orbital (Fig. 5a). In Fig. 5c, d, simulated STM images are shown of orbitals $\alpha$ and $\beta$. We considered an extended $s$-like wave function for the tip to simulate the STM appearance of orbitals $^{38,44}$. The experimentally observed image concurs with the simulated LUMO ( $\beta$ orbital) STM image of the molecule in the closed-shell resonance structure, indicating that IF is in the closed-shell configuration.

In addition, we carried out a bond-order analysis by AFM, which can be used to investigate the contributions of open- and closed-shell resonant structures, as these resonant structures lead to qualitatively different bond-order relations in the $s$-indacene moiety. The bond-order analysis of IF on $2 \mathrm{ML} \mathrm{NaCl}$ also shows excellent agreement with the closed-shell configuration. Figure 6e and $f$ shows bond orders derived from the relaxed geometries using Pauling's empirical model ${ }^{29,45-47}$. In experiment, we compare the brightness $(\Delta f)$ and apparent lengths of bonds to deduce bond-order relations $\mathrm{s}^{28,29,47}$. Bonds of greater bond order are imaged more brightly owing to greater repulsive forces and they appear shorter ${ }^{49}$. In the high-resolution AFM image on 2 $\mathrm{ML} \mathrm{NaCl}$ shown in Fig. 6c, we compared bonds that show a very similar chemical environment, i.e. bond $a$ with $b$ and bond $c$ with $d$, respectively. For IF on $\mathrm{NaCl}$ (Fig. $6 c$, d) the AFM
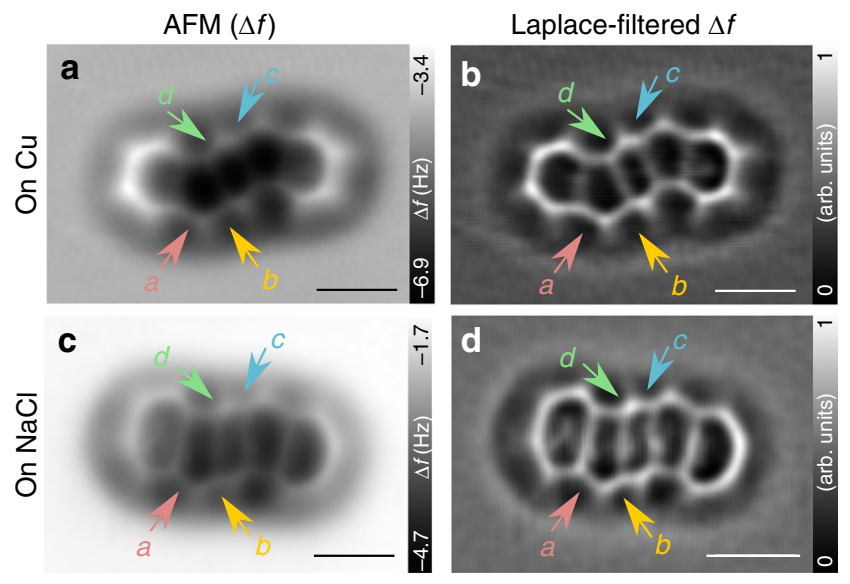

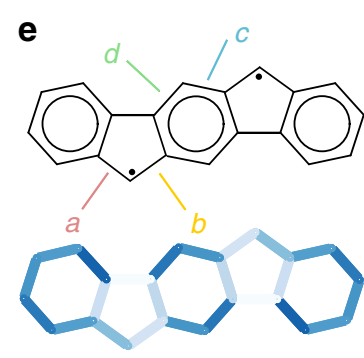

Open-shell f

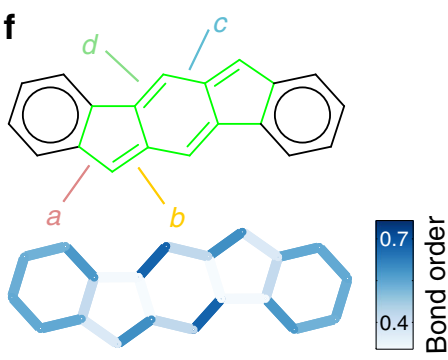

Closed-shell
Fig. 6 Bond-order analyses. a, c Constant-height AFM images of IF taken with CO tip at $V=0 \mathrm{~V}$ on $\mathrm{Cu}(111)(\Delta z=-2.1 \AA)(\mathbf{a})$ and on $2 \mathrm{ML} \mathrm{NaCl}(\Delta z$ $=1.5 \AA$ ) (c). b, d To emphasise the structure of the molecule, Laplacefiltered AFM images are also shown. e, $\mathbf{f}$ Bond orders of IF in its open-shell (e) and closed-shell (f) resonance structures. The s-indacene moiety is highlighted in green (f). On the bond-order scale, 0 refers to a single bond and 1 to a double-bond ${ }^{45}$. Bond orders were determined by using the bond lengths of relaxed geometries. Scale bars, $500 \mathrm{pm}$

measurements indicate greater bond order of $b$ than of $a$ and greater bond order of $d$ than of $c$, both in line with the closedshell configuration of IF. In our calculations, the energy of the closed-shell configuration is by $0.92 \mathrm{eV}$ lower than that of the open-shell for the gas-phase molecule. Our findings are in good agreement with the previously calculated low diradical character for $\mathrm{IF}^{12}$.

$\mathrm{On} \mathrm{Cu}(111)$, we were not able to probe the frontier molecular orbitals because of the strong electronic coupling between the molecule and the metal substrate ${ }^{42}$. However, bond-order analysis by AFM can be used to gain information about the resonance character of IF. AFM images appear less distorted on $\mathrm{Cu}(111)$ then on $\mathrm{NaCl}^{48}$. On $\mathrm{Cu}(111)$, bonds $a$ and $b$ appear with similar contrast and also their apparent bond lengths are comparable as well (Fig. 6a, b). On $\mathrm{Cu}(111)$, bond $c$, located in the central benzene ring of the $s$-indacene moiety, has a brighter appearance and a shorter apparent length than bond $d$. Thus AFM indicates that bonds $a$ and $b$ have even bond orders and $c$ has a greater bond order than $d$. The bond-order relations found on $\mathrm{Cu}(111)$ match neither with the closed-shell nor with the open-shell configurations shown in Fig. 6e, f. As discussed above, the adsorption height of IF on $\mathrm{Cu}(111)$ decreases significantly with respect to its precursor 3, indicating a strong molecule-surface interaction after dehydrogenation. Reduction of the adsorption height was shown for olympicene when the physisorbed state was changed into a chemisorbed configuration by transforming the molecule into a $\pi$-radical by atomic manipulation ${ }^{28}$. Similarly, we attribute the origin of the reduced 
adsorption height and bond-order reorganisation to chemisorption.

In conclusion, we have shown a successful on-surface and insolution generation and characterisation of highly reactive antiaromatic indeno[1,2- $b]$ fluorene (IF). We generated IF by iodide-induced debromination in solution, whereas on surface we used tip-induced dehydrogenation. In solution, we found that IF survives for a few minutes even at ambient conditions. On surface, the molecule shows its antiaromatic, closed-shell configuration on $2 \mathrm{ML} \mathrm{NaCl}$. This is in contrast to IF adsorbed on $\mathrm{Cu}(111)$, where bond-order analysis indicates significant deviations from the closed-shell configuration, demonstrating the importance of molecule-surface interactions on the $\pi$-electron distribution.

\section{Methods}

AFM/STM experiments. Experiments were carried out using a home-built combined STM/AFM under ultrahigh vacuum conditions (below $10^{-10} \mathrm{mbar}$ ) at a temperature of $5 \mathrm{~K}$. The bias voltage $V$ was applied to the sample. A qPlus sensor ${ }^{49}$ (stiffness $k=1800 \mathrm{~N} / \mathrm{m}$, eigenfrequency $f_{0}=25 \mathrm{kHz}$, quality factor $Q=2 \times 10^{5}$ ) operated in frequency-modulation mode ${ }^{50}$ was used to perform AFM measurements. A focused ion beam setup was used to cut and sharpen the PtIr tip. The oscillation amplitude was $0.5 \AA$. A $\mathrm{Cu}(111)$ single crystal was cleaned by several sputtering and annealing cycles. Ultrathin $\mathrm{NaCl}$ films were grown on $\mathrm{Cu}(111)$ by thermal evaporation of $\mathrm{NaCl}$ at a temperature of about $270 \mathrm{~K}$. Low coverages of 3 and $\mathrm{CO}$ molecules were deposited while the sample temperature was kept below 10 $\mathrm{K}$. CO tips were prepared by picking up a single $\mathrm{CO}$ molecule from $\mathrm{NaCl}^{21}$

DFT calculations. DFT calculations were performed using the FHI-AIMS code ${ }^{51}$. The geometry of the isolated molecule was optimised with the tight basis defaults. For structural relaxation, the Perdew-Burke-Ernzerhof exchange-correlation functional was applied ${ }^{52}$ with vdW correction ${ }^{53}$. The convergence criterion for the total forces was $10^{-3} \mathrm{eV} / \AA$, and for the total energy it was set to $10^{-5} \mathrm{eV}$. The closed-shell configuration was calculated by performing unrestricted spin-polarised energy minimisation or spin-unpolarised calculations. The open-shell configuration was considered by keeping a spin multiplicity of 3 for the total spin of the molecule during the spin-polarised total energy minimisation.

Hybrid functional Heyd, Scuseria and Ernzerho (HSE) ${ }^{54,55}$ with a mixing coefficient of 0.3 was applied for the computational calculations of the HOMO-LUMO gaps presented in Table 1. The mixing coefficient was adjusted to have the best match between the optical gap and the calculated gap of 6,12-mesitylIF and 2. The convergence criterion for the total forces was $10^{-3} \mathrm{eV} / \AA$, and for the total energy it was set to $10^{-5} \mathrm{eV}$.

In-solution lifetime measurements. UV/Vis spectra were recorded in a Jasco V630 spectrophotometer. See Supplementary Methods for more details about insolution experiments and the synthesis of the IF precursors 2 and 3.

Data availability. All experimental and theoretical data presented here are available from the corresponding authors on reasonable request.

Received: 30 June 2017 Accepted: 2 February 2018

Published online: 22 March 2018

\section{References}

1. Krygowski, T., Cyrañski, M., Czarnocki, Z., Häfelinger, G. \& Katritzky, A. R. Aromaticity: a theoretical concept of immense practical importance. Tetrahedron 56, 1783-1796 (2000).

2. Peeks, M. D., Claridge, T. D. W. \& Anderson, H. L. Aromatic and antiaromatic ring currents in a molecular nanoring. Nature 541, 200-203 (2017).

3. Hückel, E. Quantentheoretische Beiträge zum Benzolproblem. Z. Phys. 70, 204-286 (1931).

4. Zhao, L., Grande-Aztatzi, R., Foroutan-Nejad, C., Ugalde, J. M. \& Frenking, G. Aromaticity, the Hückel $4 n+2$ rule and magnetic current. Chem. Sel. 2, 863-870 (2017).

5. Clar, E. The Aromatic Sextet (John Wiley \& Sons, London, 1972)

6. Randić, M. Aromaticity and conjugation. J. Am. Chem. Soc. 99, 444-450 (1977).

7. Breslow, R., Brown, J. \& Gajewski, J. J. Antiaromaticity of cyclopropenyl anions. J. Am. Chem. Soc. 89, 4383-4390 (1967).
8. Zeng, Z. et al. Pro-aromatic and anti-aromatic $\pi$-conjugated molecules: an irresistible wish to be diradicals. Chem. Soc. Rev. 44, 6578-6596 (2015).

9. Frederickson, C. K., Rose, B. D. \& Haley, M. M. Explorations of the indenofluorenes and expanded quinoidal analogues. Acc. Chem. Res. 50, 977-987 (2017).

10. Tobe, Y. Non-alternant non-benzenoid aromatic compounds: past, present, and future. Chem. Rec. 15, 86-96 (2015).

11. Kubo, T. Recent progress in quinoidal singlet biradical molecules. Chem. Lett 44, 111-112 (2015)

12. Fukuda, K., Nagami, T., Fujiyoshi, J. \& Nakano, M. Interplay between openshell character, aromaticity, and second hyperpolarizabilities in indenofluorenes. J. Phys. Chem. A 119, 10620-10627 (2015).

13. Rose, B. D. et al. Experimental and computational studies of the neutral and reduced states of indeno[1,2-b]fluorene. J. Am. Chem. Soc. 136, 9181-9189 (2014).

14. Chase, D. T., Fix, A. G., Rose, B. D. \& Weber, C. D. Electron accepting 6,12 diethynylindeno $[1,2-b]$ fluorenes: synthesis, crystal structures, and photophysical properties. Angew. Chem. Int. Ed. 50, 11103-11106 (2011).

15. Rudebusch, G. E. et al. Diindeno-fusion of an anthracene as a design strategy for stable organic biradicals. Nat. Chem. 8, 753 (2016)

16. Nishida, J.-i., Tsukaguchi, S. \& Yamashita, Y. Synthesis, crystal structures, and properties of 6,12-diaryl-substituted indeno[1,2-b]fluorenes. Chem. Eur. J. 18, 8964-8970 (2012)

17. Frederickson, C. K., Zakharov, L. N. \& Haley, M. M. Modulating paratropicity strength in diareno-fused antiaromatics. J. Am. Chem. Soc. 138, 16827-16838 (2016).

18. Chase, D. T. et al. 6,12-Diarylindeno[1,2-b]fluorenes: syntheses, photophysics, and ambipolar OFETs. J. Am. Chem. Soc. 134, 10349-10352 (2012).

19. Anthony, J. E. The larger acenes: versatile organic semiconductors. Angew. Chem. Int. Ed. 47, 452-483 (2008).

20. Liu, J. et al. $\pi$-Extended and curved anti-aromatic polycyclic hydrocarbons. $J$. Am. Chem. Soc. 139, 7513-7521 (2017).

21. Gross, L., Mohn, F., Moll, N., Liljeroth, P. \& Meyer, G. The chemical structure of a molecule resolved by atomic force microscopy. Science 325, 1110-1114 (2009).

22. Lauhon, L. J. \& Ho, W. Single-molecule chemistry and vibrational spectroscopy: pyridine and benzene on $\mathrm{Cu}(001)$. J. Phys. Chem. A 104, 2463-2467 (2000).

23. Hla, S.-W., Bartels, L., Meyer, G. \& Rieder, K.-H. Inducing all steps of a chemical reaction with the scanning tunneling microscope tip: towards single molecule engineering. Phys. Rev. Lett. 85, 2777-2780 (2000).

24. Zhao, A. et al. Controlling the Kondo effect of an adsorbed magnetic ion through its chemical bonding. Science 309, 1542-1544 (2005).

25. Baadji, N. et al. Controlled sequential dehydrogenation of single molecules by scanning tunneling microscopy. Phys. Rev. B 82, 115447 (2010)

26. Mohn, F. et al. Reversible bond formation in a gold-atom-organic-molecule complex as a molecular switch. Phys. Rev. Lett. 105, 266102 (2010).

27. Lit, J. et al. Suppression of electron-vibron coupling in graphene nanoribbons contacted via a single atom. Nat. Commun. 4, 2023 (2013).

28. Schuler, B. et al. Adsorption geometry determination of single molecules by atomic force microscopy. Phys. Rev. Lett. 111, 086101 (2013).

29. Pavliček, N. et al. On-surface generation and imaging of arynes by atomic force microscopy. Nat. Chem. 7, 623-628 (2015).

30. Schuler, B. et al. Reversible Bergman cyclization by atomic manipulation. Nat Chem. 8, 220-224 (2016).

31. Gross, L. et al. Organic structure determination using atomic-resolution scanning probe microscopy. Nat. Chem. 2, 821-825 (2010).

32. Kawai, S. et al. Thermal control of sequential on-surface transformation of a hydrocarbon molecule on a copper surface. Nat. Commun. 7, 12711 (2016).

33. Rogers, C. et al. Closing the nanographene gap: surface-assisted synthesis of peripentacene from 6,6-bipentacene precursors. Angew. Chem. Int. Ed. 54, 15143 (2015).

34. de Oteyza, D. G. et al. Direct imaging of covalent bond structure in singlemolecule chemical reactions. Science 340, 1434-1437 (2013).

35. Kawai, S. et al. Competing annulene and radialene structures in a single antiaromatic molecule studied by high-resolution atomic force microscopy. ACS Nano 11, 8122-8130 (2017).

36. Cava, M. P. \& Napier, D. R. Condensed cyclobutane aromatic systems. II. Dihalo derivatives of benzocyclobutene and benzocyclobutadiene dimer. I. Am. Chem. Soc. 79, 1701-1705 (1957).

37. Segura, J. L. \& Martín, N. o-Quinodimethanes: efficient intermediates in organic synthesis. Chem. Rev. 99, 3199-3246 (1999).

38. Pavliček, N. et al. Generation and characterization of triangulene. Nat. Nanotechnol. 12, 308-311 (2017).

39. Majzik, Z. et al. Synthesis of a naphthodiazaborinine and its verification by planarization with atomic force microscopy. ACS Nano 10, 5340-5345 (2016). 
40. McMahon, T. B. \& Kebarle, P. Intrinsic acidities of carbon and nitrogen acids measured by gas phase proton transfer equilibria. Substituent effects on the stabilities of gas phase carbanions and nitrogen anions. J. Am. Chem. Soc. 98, 3399-3406 (1976).

41. Swart, I., Sonnleitner, T. \& Repp, J. Charge state control of molecules reveals modification of the tunneling barrier with intramolecular contrast. Nano Lett. 11, 1580-1584 (2011).

42. Repp, J., Meyer, G., Stojković, S. M., Gourdon, A. \& Joachim, C. Molecules on insulating films: scanning-tunneling microscopy imaging of individual molecular orbitals. Phys. Rev. Lett. 94, 086101 (2005).

43. Bennewitz, R. et al. Aspects of dynamic force microscopy on $\mathrm{NaCl} / \mathrm{Cu}(111)$ : resolution, tip-sample interactions and cantilever oscillation characteristics. Surf. Interface Anal. 27, 462-466 (1999).

44. Pavliček, N., Swart, I., Niedenführ, J., Meyer, G. \& Repp, J. Symmetry dependence of vibration-assisted tunneling. Phys. Rev. Lett. 110, 136101 (2013).

45. Pauling, L., Brockway, L. O. \& Beach, J. Y. The dependence of interatomic distance on single bond-double bond resonance. J. Am. Chem. Soc. 57, 2705-2709 (1935).

46. Sedlar, J., Anelić, I., Gutman, I., Vukičević, D. \& Graovac, A. Vindicating the Pauling-bond-order concept. Chem. Phys. Lett. 427, 418-420 (2006).

47. Gross, L. et al. Bond-order discrimination by atomic force microscopy. Science 337, 1326-1329 (2012).

48. Neu, M. et al. Image correction for atomic force microscopy images with functionalized tips. Phys. Rev. B 89, 205407 (2014).

49. Giessibl, F. J. Atomic resolution on $\mathrm{Si}(111)-(7 \times 7)$ by noncontact atomic force microscopy with a force sensor based on a quartz tuning fork. Appl. Phys. Lett. 76, 1470-1472 (2000).

50. Albrecht, T. R., Grütter, P., Horne, D. \& Rugar, D. Frequency modulation detection using high-Q cantilevers for enhanced force microscope sensitivity. J. Appl. Phys. 69, 668-673 (1991).

51. Blum, V. et al. Ab initio molecular simulations with numeric atom-centered orbitals. Comput. Phys. Commun. 180, 2175-2196 (2009).

52. Perdew, J. P., Burke, K. \& Ernzerhof, M. Generalized gradient approximation made simple. Phys. Rev. Lett. 77, 3865-3868 (1996).

53. Tkatchenko, A. \& Scheffler, M. Accurate molecular van Der Waals interactions from ground-state electron density and free-atom reference data. Phys. Rev. Lett. 102, 073005 (2009).

54. Krukau, A. V., Vydrov, O. A., Izmaylov, A. F. \& Scuseria, G. E. Influence of the exchange screening parameter on the performance of screened hybrid functionals. J. Chem. Phys. 125, 224106 (2006).

55. Heyd, J., Scuseria, G. E. \& Ernzerhof, M. Hybrid functionals based on a screened Coulomb potential. J. Chem. Phys. 118, 8207-8215 (2003).

\section{Acknowledgements}

We thank B. Schuler, S. Fatayer and R. Allenspach for discussions. We acknowledge financia support from the European Research Council Advanced Grant CEMAS (291194), the European Union project PAMS (610446) and the ERC Consolidator Grant AMSEL (682144) programs, the Agencia Estatal de Investigación (MAT2016-78293-C6-3-R and CTQ201678157-R), the Xunta de Galicia (Centro singular de investigación de Galicia accreditation 2016-2019, ED431G/09) and the European Regional Development Fund (ERDF).

\section{Author contributions}

Z.M., N.P., G.M. and L.G. performed and analysed the AFM/STM measurements. Z.M., N.P. and N.M. carried out the DFT calculations. M.V.-V., D.Pér., E.G. and D.Peñ. synthesised precursor molecules and performed the in-solution experiments. All authors contributed to writing the paper.

\section{Additional information}

Supplementary Information accompanies this paper at https://doi.org/10.1038/s41467 018-03368-9.

Competing interests: The authors declare no competing interests.

Reprints and permission information is available online at http://npg.nature.com/ reprintsandpermissions/

Publisher's note: Springer Nature remains neutral with regard to jurisdictional claims in published maps and institutional affiliations.

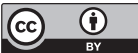

Open Access This article is licensed under a Creative Commons Attribution 4.0 International License, which permits use, sharing, adaptation, distribution and reproduction in any medium or format, as long as you give appropriate credit to the original author(s) and the source, provide a link to the Creative Commons license, and indicate if changes were made. The images or other third party material in this article are included in the article's Creative Commons license, unless indicated otherwise in a credit line to the material. If material is not included in the article's Creative Commons license and your intended use is not permitted by statutory regulation or exceeds the permitted use, you will need to obtain permission directly from the copyright holder. To view a copy of this license, visit http://creativecommons.org/ licenses/by/4.0/.

(C) The Author(s) 2018 